\title{
Modeling and experimental studies of magnetron injection locking
}

\author{
P. Pengvanich, V. B. Neculaes, ${ }^{\text {a) }}$ Y. Y. Lau, ${ }^{\text {b) }}$ R. M. Gilgenbach, M. C. Jones, ${ }^{\text {c) }}$ \\ W. M. White, and R. D. Kowalczyk ${ }^{\mathrm{d})}$ \\ Plasma, Pulsed Power and Microwave Laboratory, Department of Nuclear Engineering and Radiological \\ Sciences, University of Michigan, Ann Arbor, Michigan 48109-2104
}

(Received 13 June 2005; accepted 7 October 2005; published online 5 December 2005)

\begin{abstract}
A phase-locking model has been developed from circuit theory to qualitatively explain the various regimes observed in magnetron injection-locking experiments. The experiments utilize two continuous-wave oven magnetrons: one functions as an oscillator and the other as a driver. The model includes both magnetron-specific electronic conductance and frequency-pulling parameter. Both time and frequency domain solutions are developed from the model, allowing investigations into the growth and saturation as well as the frequency response of the output signal. This simplified model recovers qualitatively many of the phase-locking frequency characteristics observed in the experiments. @ 2005 American Institute of Physics. [DOI: 10.1063/1.2132513]
\end{abstract}

\section{INTRODUCTION}

Phase locking is utilized today in many important applications, ranging from small-scale devices such as cardiac pacemakers ${ }^{1}$ to large-scale devices such as radar. $^{2}$ In the development of high-power microwave sources, phase locking of relativistic magnetrons has been extensively studied. ${ }^{3-13}$ Some of these experiments were designed to combine the power of several relativistic magnetrons in a phase-locked array. ${ }^{3,4}$ A more recent experiment used a lower power but more stable magnetron to control a high-power relativistic magnetron that exhibits mode competition. ${ }^{5}$ Performance of the pulsed relativistic magnetrons could improve if priming by an external signal exclusively excites the desired mode, usually the pi mode. Recently, interest in phase locking of nonrelativistic magnetrons was renewed due to its possible application in the solar power satellite (SPS) ${ }^{14}$ The availability, efficiency, low cost, size, ruggedness, and reliability of the oven magnetrons make them very attractive as a frequency injection-locked amplifier for the SPS. ${ }^{15}$

In this paper, we present the theory and experiments on frequency locking using two continuous-wave (cw) oven magnetrons. The analytical model closely follows $\mathrm{Chen}^{6}$ who made use of the Adler condition ${ }^{16}$ and the van der Pol equation $^{17}$ but included magnetron-specific growthsaturation characteristic ${ }^{18}$ and nonlinear frequency-pulling effect. ${ }^{19}$ The latter is believed to be especially important for relativistic magnetrons. While Chen constructed the model for relativistic magnetrons, we adopt it for the cw kilowatt oven magnetron experiments. ${ }^{20}$

In Sec. II, a general phase-locking theory for magnetron is presented. Both magnetron-specific effects mentioned above are included in the derivation. Numerical results with discussions on a low-power injection-locking application are presented toward the end of Sec. II. Experimental injection

\footnotetext{
a) Present address: General Electric Global Research, Niskayuna, NY.

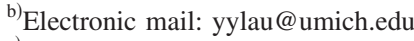

${ }^{c}$ Present address: Sandia National Laboratory, Albuquerque, NM.

${ }^{d)}$ Present address: L-3 Communications, San Carlos, CA.
}

locking with $\mathrm{cw}$ oven magnetrons ${ }^{20}$ is presented in Secs. III and IV. In Sec. V, we compare the numerical and experimental results.

\section{PHASE-LOCKING THEORY AND NUMERICAL SIMULATIONS FOR MAGNETRON}

What sets the magnetron apart from other types of oscillators is that the electrons are born and interact with both dc and rf electric fields inside a common resonant cavity. The single-mode equivalent-circuit model for the magnetron shown in Fig. 1 consists of (a) the RLC circuit which represents the resonance cavity itself, (b) the electronic conductance $g$ and electronic susceptance $b$ which account for the dc-electron and rf-electron interactions inside the cavity, and (c) the load conductance $G$ and load susceptance $B$ which represent the admittance looking into an external load. ${ }^{6}$

Current conservation of the circuit in Fig. 1 can be written as

$$
-(g+j b) \widetilde{V}_{\mathrm{rf}}=\frac{\tilde{V}_{\mathrm{rf}}}{R}+\frac{\tilde{V}_{\mathrm{rf}}}{j \omega L}+j \omega C \widetilde{V}_{\mathrm{rf}}+C \omega_{0} \frac{(G+j B)}{Q_{\mathrm{ext}}} \widetilde{V}_{\mathrm{rf}},
$$

where $\omega_{0}=(L C)^{-1 / 2}$ is the resonance frequency of the unloaded cavity, $Q_{\text {ext }}$ is the quality factor of the external load, and $\widetilde{V}_{\text {rf }}$ is the output rf voltage containing fast and slow temporal components. The fast temporal component of $\widetilde{V}_{\text {rf }}$ has $e^{j \omega t}$ dependence so that $\widetilde{V}_{\mathrm{rf}}=V_{\mathrm{rf}}(t) \cos (\omega t)$, where $V_{\mathrm{rf}}(t)$ is slowly varying with a time rate much smaller than $\omega$. For magnetrons, $g$ and $b$ have been suggested ${ }^{6}$ to obey the relations $g=-(1 / R)\left(V_{\mathrm{dc}} / V_{\mathrm{rf}}-1\right)$ and $b=b_{0}+g \tan \alpha$, where $V_{\mathrm{dc}}$ is the dc voltage across the $A-K$ gap, $b_{0}$ is a constant, and $\alpha$ is known as the frequency-pushing parameter which is typically on the order of unity. Figure 2 qualitatively shows $-g$ and $-b$ as functions of $V_{\mathrm{rf}}$. In this model, the negative slope of $-g$ is responsible for the magnetron growth and saturation characteristics. ${ }^{6,18}$ 


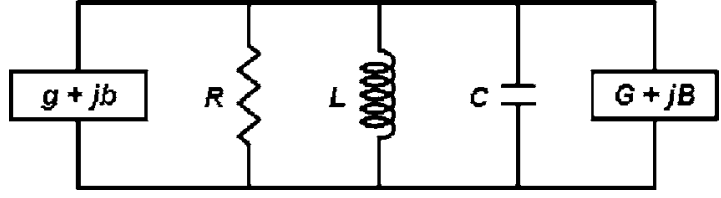

FIG. 1. The circuit model.

Steady-state analytic solutions of (1) can be obtained by assuming that $V_{\text {rf }}$ is constant, separating the equation into real and imaginary parts, and solving for $V_{\mathrm{rf}}$ and $\omega$, which is real. The normalized results then are ${ }^{6}$

$$
\begin{aligned}
& V_{\mathrm{rf}, \text { sat }}=\frac{Q_{L}}{Q_{L}+Q_{0}}, \\
& \omega_{\mathrm{sat}} \equiv \omega^{\prime}=\sqrt{1-\frac{b_{0}}{Q_{0}}-\frac{B}{Q_{\mathrm{ext}}}-\frac{\tan \alpha}{Q_{L}}},
\end{aligned}
$$

where $1 / Q_{L}=1 / Q_{0}+G / Q_{\text {ext }}$ and $Q_{0}=\omega_{0} R C$. In deriving (2a) and (2b), the voltage is normalized by $V_{\mathrm{dc}}$, time by $1 / \omega_{0}$, frequency by $\omega_{0}$, and admittance by $1 / R$. These normalizations will be used hereafter unless otherwise specified. For simplicity, $\omega / \omega_{0}$ is also assumed to be roughly unity. The details of the derivation along with the approximate temporal solution of $V_{\text {rf }}$ can be found in the work of Chen. ${ }^{6}$

When an external source of current $\tilde{i}_{1}$ and voltage $\widetilde{V}_{1}$ is applied to drive the magnetron, the load admittance $Y_{\text {load }}$ is modified accordingly: ${ }^{18}$

$$
Y_{\text {load }}=\frac{\tilde{i}_{\mathrm{rf}}+\tilde{i}_{1}}{\tilde{V}_{\mathrm{rf}}+\tilde{V}_{1}},
$$

where $\tilde{i}_{\mathrm{rf}}$ and $\widetilde{V}_{\mathrm{rf}}$ are, respectively, the complex current and voltage delivered to the magnetron at its plane of reference. For convenience of notation, we will assume that the magnetron is driven by an external current source and let $\widetilde{V}_{1}=0$. (The last expression on the right side of Eq. (4a) below will still be valid even when $\widetilde{V}_{1}$ has a nonzero value. Equation (4b) needs to be modified accordingly ${ }^{18}$ in which case $\rho$ still represents the relative amplitudes of the external and the rf signals.) Equation (3) then reads

$$
Y_{\text {load }}=\frac{\tilde{i}_{\mathrm{rf}}}{\tilde{V}_{\mathrm{rf}}}+\frac{\tilde{i}_{1}}{\tilde{V}_{\mathrm{rf}}}=(G+i B)+\rho e^{j \theta},
$$

where

$$
\rho=i_{1} / V_{\mathrm{rf}},
$$

and $\theta$ is the relative phase difference between the phase of the external driving signal and the phase of the rf output. Hence, assuming that the phase of the external driving signal is $\Phi_{1}=\omega_{1} t$, the phase of the output signal would be $\Phi_{0}$ $=\omega_{1} t-\theta$. Current conservation yields

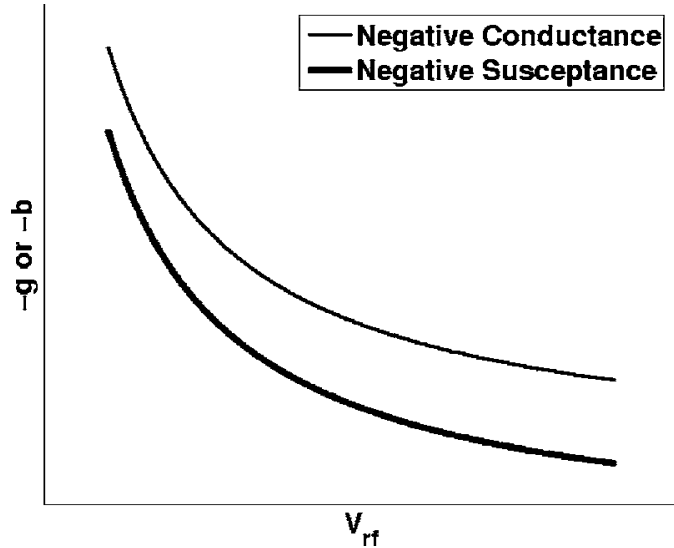

FIG. 2. $g$ and $b$ as functions of $V_{\mathrm{rf}}$.

$$
\begin{aligned}
-(g+j b) \tilde{V}_{\mathrm{rf}}= & \frac{\tilde{V}_{\mathrm{rf}}}{R}+\frac{\tilde{V}_{\mathrm{rf}}}{j \omega L}+j \omega C \tilde{V}_{\mathrm{rf}} \\
& +C \omega_{0} \frac{(G+j B)+\rho e^{j \theta}}{Q_{\mathrm{ext}}} \widetilde{V}_{\mathrm{rf}}
\end{aligned}
$$

where $\widetilde{V}_{\text {rf }}$ is now of the form $\tilde{V}_{\mathrm{rf}}=V_{\mathrm{rf}}(t) \cos \left[\omega_{1} t-\theta(t)\right]$.

By allowing both $V_{\mathrm{rf}}$ and $\theta$ to slowly vary in time, Eq. (5) can be decoupled into two normalized first-order slowly time-varying equations: ${ }^{6}$

$$
\begin{aligned}
& \frac{d \theta}{d t}+1-\omega_{1}=\frac{\rho}{2 Q_{\mathrm{ext}}} \sin \theta, \\
& \frac{1}{V_{\mathrm{rf}}} \frac{d V_{\mathrm{rf}}}{d t}+\frac{1}{Q_{0}}\left(1-\frac{1}{V_{\mathrm{rf}}}\right)=-\frac{\rho}{2 Q_{\mathrm{ext}}} \cos \theta .
\end{aligned}
$$

Consequently, the free-running magnetron oscillates at its cold resonance frequency of $\omega^{\prime}=1$. The rf voltage $V_{\mathrm{rf}}$ in Eqs. (6a) and (6b) has been rescaled so that its undriven, saturation value is unity. Since $\rho$ has $V_{\text {rf }}$ dependence, these coupled equations govern the amplitude and phase evolution during the lock-in process. The locking condition can be analytically solved from (6a) by setting $d \theta / d t=0$. This gives

$$
1-\omega_{1}=\frac{\rho}{2 Q_{\mathrm{ext}}} \sin \theta
$$

or

$$
2 Q_{\text {ext }}\left|1-\omega_{1}\right| \leqslant \rho,
$$

which is the well-known Adler condition. ${ }^{16,18}$ The phase shift near locking can be obtained by pretending $\rho=$ constant and rewriting (6a) as

$$
\frac{d \theta}{d t}=\frac{\rho}{2 Q_{\mathrm{ext}}} \sin \theta+\left(\omega_{1}-1\right),
$$

whose explicit solution is ${ }^{18}$

$$
\tan \left(\frac{\theta}{2}\right)=\frac{A}{B}-F \sqrt{\left(\frac{A}{B}\right)^{2}-1,}
$$

where 


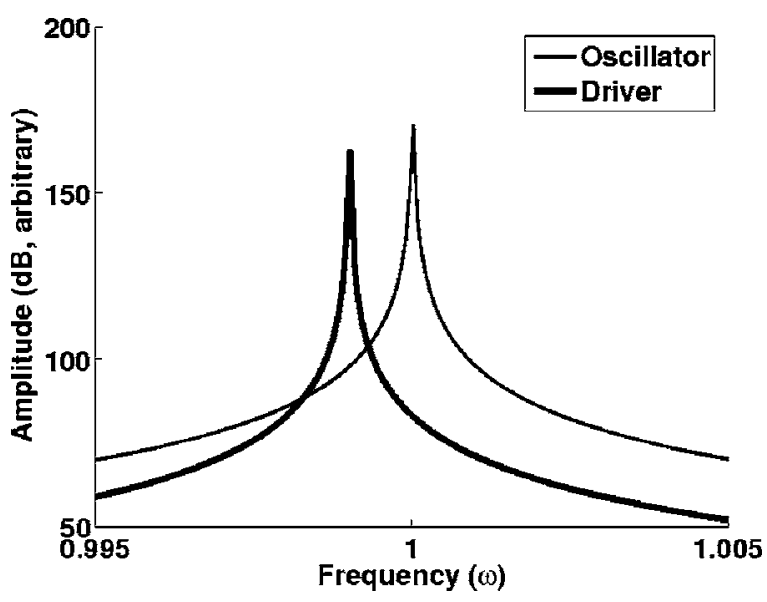

FIG. 3. Free-running oscillator and external driving signals.

$$
\begin{aligned}
& A=\frac{\rho}{2 Q_{\mathrm{ext}}}, \\
& B=1-\omega_{1}, \\
& D=\sqrt{A^{2}-B^{2}},
\end{aligned}
$$

and

$$
F=\frac{1+e^{D\left(t-t_{0}\right)}}{1-e^{D\left(t-t_{0}\right)}} .
$$

There are three regions of interest.

(i) $\quad D$ is real. In this case, (8) is satisfied and the magnetron is phase locked to the external source. As time increases, $F$ approaches -1 , and $\theta$ has a constant value which can be easily determined by solving (10). It can be shown that when (8) is marginally satisfied, the phase shift between the magnetron and the external source is $\pi / 2$.

(ii) $\quad D$ is small and imaginary. In this case, the magnetron is not phase locked to the external source. We can write $F=i \cot \left(|D|\left(t-t_{0}\right) / 2\right)$ such that the right-hand side of (10) becomes periodic with a period of $2 \pi /|D| . \theta$ is no longer a constant but is a superposition between a linear function of time and a function with periodicity $2 \pi /|D|$. Therefore, sideband frequencies at integral multiples of $|D| / 2 \pi$ can be expected in this case. As $\rho$ increases, the periodicity becomes smaller, and the sidebands are expected to move closer to $\omega_{1}$.

(iii) $\quad D$ is large and imaginary. In this case, $B \gg A$, and the right-hand side of (10) becomes $\cot \left(|D|\left(t-t_{0}\right) / 2\right)$. Thus, $\theta$ becomes a linear function of time. The oscillating frequency of the magnetron is therefore unaffected by the source frequency.

Equations (6a) and (6b) can be numerically solved for $V_{\text {rf }}$ and $\theta$ using the Runge-Kutta method. ${ }^{21,22}$ A matLaB $^{\circledast}$ (Ref. 23) algorithm was written to numerically solve (6a) and (6b) for $V_{\mathrm{rf}}$ and $\theta$. The external driving signal $\rho$ is applied after a specific time, e.g., after the free-running signal saturates, to mimic the cw "injection-locking" experiment. The output

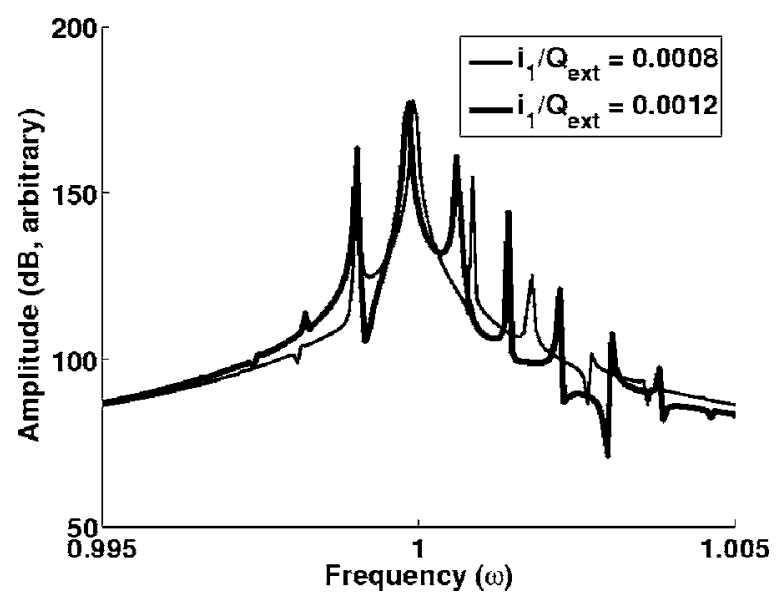

FIG. 4. Locking signal at $i_{1} / Q_{\mathrm{ext}}=0.0008$ and 0.0012 .

signal $\tilde{V}_{\text {rf }}$ including both amplitude and phase dependence can then be reconstructed. Its power spectrum is analyzed using fast Fourier transform.

Figure 3 shows the power spectra of the free-running signal constructed from (6a) and (6b) using the initial conditions $V_{\mathrm{rf}}(0)=0.001$ and $\theta(0)=0$. Also shown in Fig. 3 is the spectrum of the drive signal that is to be applied after the free-running signal reaches its steady state. The center frequency of the free-running signal is at $\omega_{0}=1$, and it is to be locked to the external driving signal at $\omega_{1}=0.999$. According to Eqs. (8) and (4b), with the free-running $V_{\text {rf }}$ normalized to unity, locking with these frequencies occurs when $i_{1} / Q_{\text {ext }}$ $\geqslant 0.002$. Figures 4 and 5 show the power spectra of $\tilde{V}_{\text {rf }}$ at various $i_{1} / Q_{\text {ext }}$. When $i_{1} / Q_{\text {ext }}$ is much lower than 0.002 , the magnetron frequency is unaffected by the driving frequency, and the power spectrum has a dominant peak at $\omega_{0}=1$ as in the free-running case and some sidebands at multiple integers of 0.001 , which is equivalent to the difference between $\omega_{0}$ and $\omega_{1}$, away from the $\omega_{0}=1$ peak. This is similar to the aforementioned case (ii) when $D$ is small and imaginary. As $i_{1} / Q_{\text {ext }}$ approaches the locking criterion, the sidebands become stronger while the dominant peak becomes smaller. The frequency separation between adjacent sidebands also becomes smaller. When locking occurs, the sidebands disap-

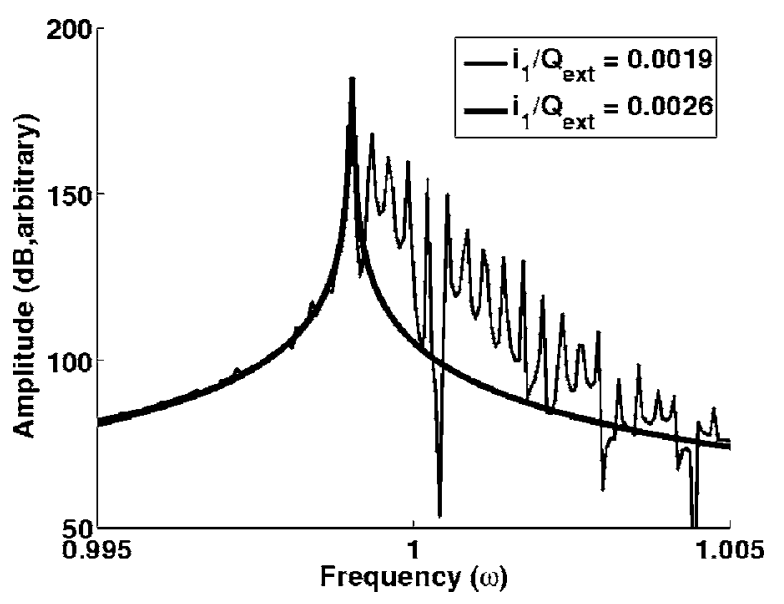

FIG. 5. Locking signal at $i_{1} / Q_{\mathrm{ext}}=0.0019$ and 0.0026 . 


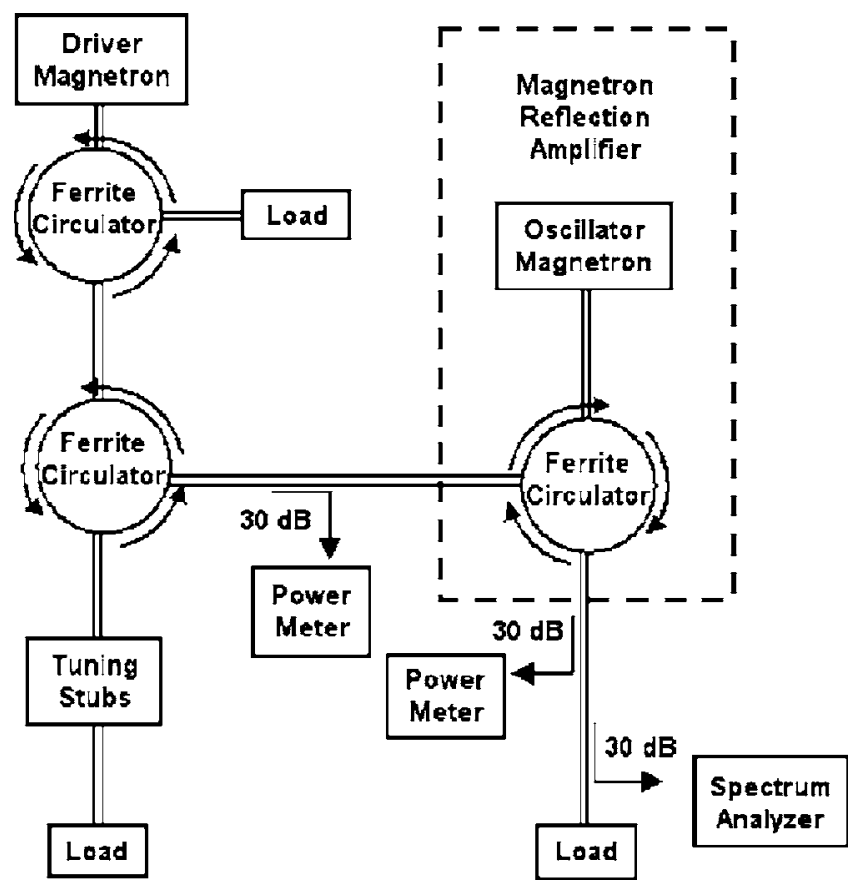

FIG. 6. The reflection amplifier setup.

pear and the oscillator oscillates at the frequency centered around $\omega_{1}$ as predicted by case (i) when $D$ becomes real.

We have observed that phase locking may occur even when the Adler condition, Eq. (8), is not met. In such cases, a closer examination of $V_{\mathrm{rf}}$ in time domain shows that $V_{\mathrm{rf}}$ violently fluctuates when the external driving signal is initially applied, before it settles into a new saturation level which is lower than the saturation level in the free-running case. We suspect that the initial fluctuation allows phase locking to occur at a drive level below the Adler criterion. This interesting topic is beyond the scope of this paper.

\section{EXPERIMENTAL SETUP}

Two cw 2.45-GHz 800-W magnetrons are used by the microwave research group at the University of Michigan ${ }^{20}$ to demonstrate phase locking in reflection amplifier experiments. One magnetron functions as a driver and the other as an oscillator. The experimental configuration is shown in Fig. 6. The driver magnetron is manufactured by National Electronics, model SXRH (with ASTEX power supply, model $\mathrm{S}$-1000i). The oscillator magnetron is manufactured by National Electronics, model HS (with ASTEX power supply, model S-1000). These ASTEX power supplies are very stable and can deliver a well-filtered dc voltage. Three waveguide circulators are used to separate the direct and reflected powers so that the two magnetrons are not mutually coupled. The majority of the microwave power produced by the driver magnetron is dissipated into a water load, while a controlled fraction is injected into the oscillator magnetron. A three-stub tuner is employed for the purpose of varying the amount of power injected into the oscillator without changing the injected frequency. Several $30 \mathrm{~dB}$ directional couplers are implemented in order to sample microwave signals for power measurement (with Agilent E4418B digital power

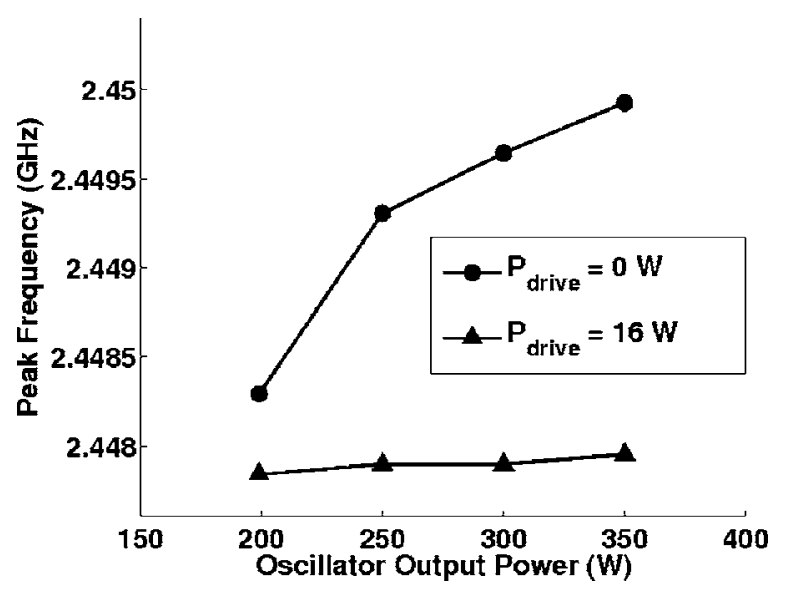

FIG. 7. Peak frequency dependence on the output power of the free-running oscillator (zero drive power). With an external injected power at $16 \mathrm{~W}$, the oscillator frequency remains constant (locked).

meters) and spectrum measurements (with an Agilent 8564 EC spectrum analyzer). WR-284 waveguides (2.84 in. wide) have been used in experiments.

It should be mentioned that the ASTEX power supplies yield stable (in time) oven magnetron microwave spectra. The central peak in the microwave spectra (corresponding to the $2.45-\mathrm{GHz}$ pi-mode oscillation) does not exhibit time jitter or amplitude modulation. This stability allows relatively accurate frequency and phase noise measurements. A $100-\mathrm{kHz}$ resolution bandwidth was utilized in spectrum analyzer measurements.

Magnetron filament power is controlled automatically within the power supply for optimum operation at every power level. The only control offered by the ASTEX power supplies is the microwave power level. Peak frequency is directly proportional to the output power for both magnetrons. Previously, Brown ${ }^{15}$ used a frequency-pulling section to change the driver frequency; in our experiments the driver frequency change has been achieved by varying the output power of the driver magnetron.

\section{EXPERIMENTAL RESULTS}

Initial experiments show that the oscillator magnetron peak frequency increases when the output power (current) increases as illustrated in Fig. 7. It is found that this magnetron behavior can be altered by injecting an external signal to force the output frequency to remain relatively constant. At zero drive, as the free-running oscillator output power increases from 200 to $350 \mathrm{~W}$, its peak frequency changes by $0.07 \%$. When $16-\mathrm{W}$ power from the driver is injected into the oscillator, the peak frequency remains comparatively constant and locked to the driver frequency at $2.4478 \mathrm{GHz}$.

Further detailed experiments are performed ${ }^{20}$ to understand the physics of injection locking. By fixing the driver output power, the driver frequency is maintained constant at $2.4482 \mathrm{GHz}$. The free-running oscillator produces $825 \mathrm{~W}$ of the microwave power $\left(P_{0}\right)$ with the frequency centered around $\omega_{0} / 2 \pi=2.4511 \mathrm{GHz}$. Power spectra of the oscillator 


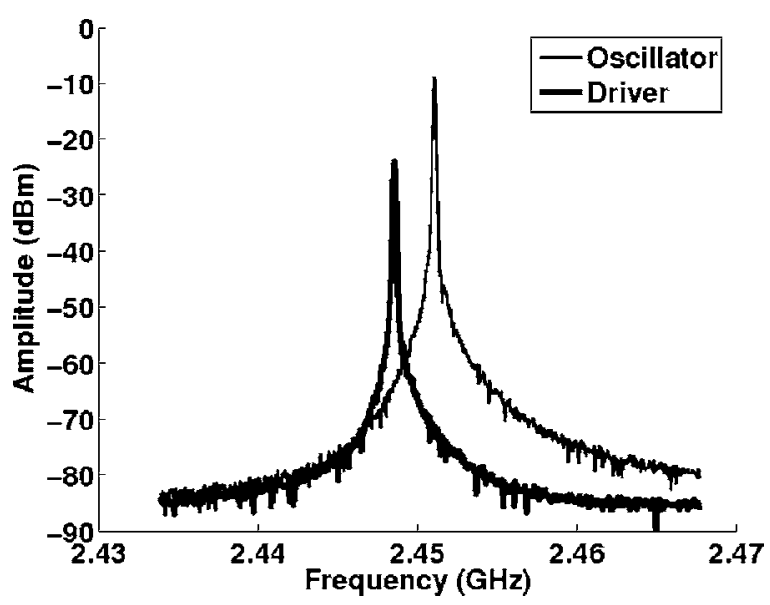

FIG. 8. The oscillator and the driver in free-running mode for the experiments performed to study the mechanism of injection locking (varied $P_{\text {drive }}$ ). $P_{0}=825 \mathrm{~W}$.

and the driver in free-running state are shown in Fig. 8. For $Q_{\text {ext }} \approx 250$, Adler's condition gives the required injected power $P_{\text {inject }}$ for phase locking: ${ }^{16}$

$$
\begin{aligned}
P_{\text {inject }} & \geqslant P_{0} Q_{\text {ext }}^{2}\left(\frac{f_{0}-f_{1}}{f_{0}}\right)^{2} \\
& =(825)(250)^{2}\left(\frac{2.4511-2.4482}{2.4511}\right)^{2} \approx 58 \mathrm{~W} .
\end{aligned}
$$

The injected power can be varied without changing the driver frequency by adjusting the (reflecting) three-stub tuner. Figures 9 and 10 present various stages of injection locking as the injected power is increased. For the injected power of $5 \mathrm{~W}$, the spectrum already shows dramatic changes from the free-running state. While the main peak of the reflection amplifier spectrum has roughly the same frequency as the free-running oscillator magnetron, there are sidebands situated at multiples of $3 \mathrm{MHz}(6 \mathrm{MHz}, 9 \mathrm{MHz}$, etc.) away from the carrier. These numbers correspond to the integer multiple of the frequency difference between the driver and the free-running oscillator. Therefore, even with 5-W injected power, the reflection amplifier shows the potential for injection locking.

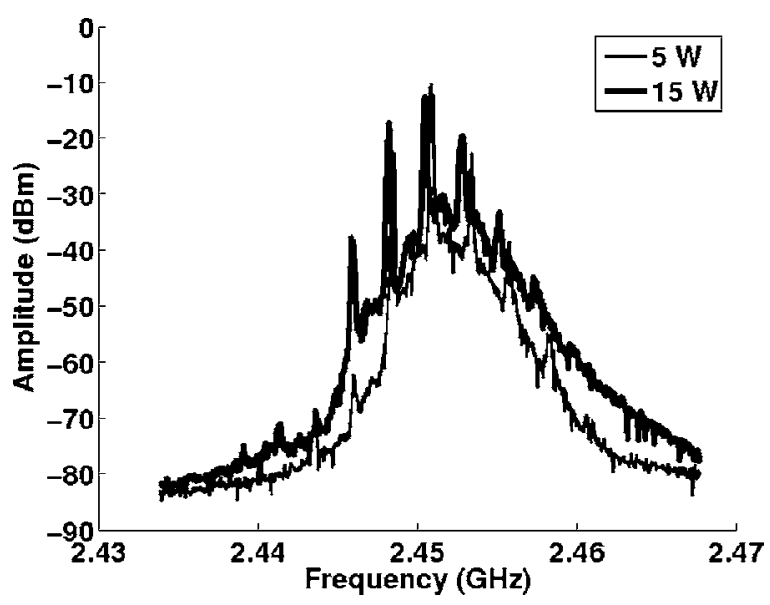

FIG. 9. Reflection amplifier microwave spectra when $P_{\text {drive }}$ are 5 and $15 \mathrm{~W}$.

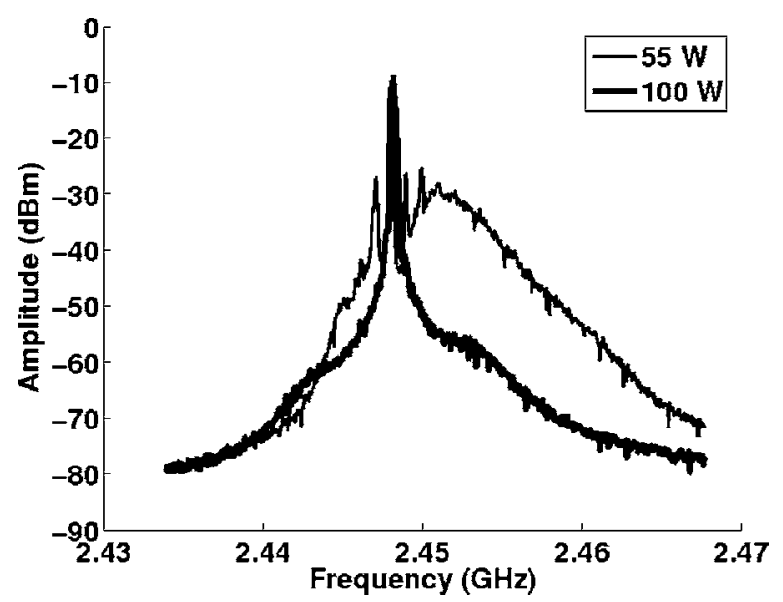

FIG. 10. Reflection amplifier microwave spectra when $P_{\text {drive }}$ are 55 and $100 \mathrm{~W}$.

As the injected power is increased to $15 \mathrm{~W}$, the height of the main peak decreases while the secondary peaks, each $3 \mathrm{MHz}$ from the carrier, gain strength. This effect is significant, and from Fig. 9 one could predict that the more power injected in the oscillator, the stronger the peak at $2.4482 \mathrm{GHz}$. One can observe that at $55-\mathrm{W}$ injected power in Fig. 10 [slightly lower than the required locking power predicted in Eq. (12)], the highest peak in the reflection amplifier microwave spectrum is emitted near $2.4482 \mathrm{GHz}$, i.e., the frequency of the driver. The oscillator frequency is therefore partially locked to the driver frequency. Despite the fact that the emitted frequency has the desired value in this case, there exist some secondary peaks. There is also a large "bump," at roughly $17 \mathrm{~dB}$ below the carrier, at frequencies above the carrier. These secondary peaks have been described and predicted by the aforementioned analytical model, specifically in case (ii) when $D$ is small and imaginary. At 100-W injected power, however, all secondary peaks disappear and the reflection amplifier frequency is completely locked at the driver frequency (Fig. 10) as predicted in case (i) when $D$ is real. Nevertheless, small plateaus on both sides of the main peak are still visible.

\section{COMPARISON BETWEEN NUMERICAL CALCULATIONS AND EXPERIMENTAL RESULTS}

In both numerical calculations (Figs. 3, 4, and 5) and in experiments (Figs. 8, 9, and 10), the externally injected signal is below the oscillator frequency by $0.1 \%$. That is, the fractional frequency change was maintained as a constant,

$$
\left(\frac{f_{0}-f_{1}}{f_{0}}\right)=0.1 \% \text {. }
$$

Frequency analyses of the oscillator output signals allow qualitative comparisons between the experimental data and the theoretical model in three regimes of phase locking: no locking, partial locking, and full locking. No locking indicates that the oscillator frequency is slightly affected or unaffected by the driver frequency, and therefore continues to oscillate mainly at its free-running frequency. Partial locking indicates that the oscillator tends to oscillate at the driver frequency while still also oscillating at its free-running fre- 
quency. Full locking indicates that the oscillator fully oscillates at the driver frequency. In subsequent discussions, it is important to note that the locking frequency of the driver is lower than the free-running frequency of the oscillator, both in the experiment and the simulation. The predicted locking criterion is $P_{\text {drive }} \geqslant 58 \mathrm{~W}$ for the experiment, and $i_{1} / Q_{\text {ext }}$ $\geqslant 0.002$ for the simulation.

The following phase-locking characteristics have been observed both in the injection-locking experiment (Figs. 9 and 10) and in the simulation (Figs. 4 and 5) based upon the presented theoretical model.

(1) When $P_{\text {drive }}$ and $i_{1} / Q_{\text {ext }}$ are substantially below the locking criterion (Figs. 4 and 9), (a) the dominant peaks on all the frequency spectra are emitted near the freerunning oscillator frequency. No locking occurs and the oscillator mainly oscillates at its free-running frequency. In all cases, the strength of the dominant peaks is also lower than the strength of the free-running peaks in Fig. 3 and 8. (b) Sidebands are observed above and below the dominant frequency peak. These sidebands are emitted at the frequencies which differ from the frequency of the dominant peak roughly by multiple integers of the frequency difference between the free-running oscillator and the driver frequencies. Consequently, the first sideband below the main peak is emitted exactly at the driver frequency. The strength of the sidebands substantially reduces further away from the dominant peak. The reduction appears to be more prominent on the sidebands below the driver frequency, which is hardly surprising considering the free-running spectra in Figs. 3 and 8 .

(2) As $P_{\text {drive }}$ and $i_{1} / Q_{\text {ext }}$ are closer to Adler's locking criterion (Figs. 4 and 9), (a) the dominant peak and all sidebands above the driver frequency move toward the driver frequency, while the sidebands below the driver frequency stay at the same values. (b) The strength of the dominant frequency peak continuously subsides while the sidebands become stronger.

(3) When $P_{\text {drive }}$ and $i_{1} / Q_{\text {ext }}$ are very close to the locking criterion (Figs. 5 and 10), the sideband emitted at the driver frequency becomes the dominant peak. The oscillator frequency is partially locked to the driver frequency. The frequencies of the previous dominant peak and the other sidebands shift accordingly and cluster around the new dominant peak.

(4) Full phase locking is confirmed in both experiment and simulation when $P_{\text {drive }}$ and $i_{1} / Q_{\text {ext }}$ are above the Adler criterion (Figs. 5 and 10). All sidebands disappear leaving only the dominant peak emitted at the driver frequency. The strength of the peaks is comparable to that of the free-running oscillator peaks in Figs. 3 and 8.

Another characteristic which the injection-locking experiment manifests, but has not been captured in the theoretical model, is the spectral plateaus around the phase-locked signal shown in Fig. 10 when $P_{\text {drive }}=100 \mathrm{~W}$. It has been confirmed that the spectral plateaus continue to exist even at higher drive power.
On the other hand, the numerical simulation based on the theoretical model suggests that phase locking can occur even when the Adler locking criterion is not met. For a given drive power, this translates to some additional locking bandwidth.

It should be mentioned that although the discrepancies in the quantitative behaviors between the experimental and the simulation results may be attributed to the oversimplification of the model employed, some of them could be explained by the limitations of the spectrum analyzer used in the experiment. Such limitations include the finite sweep time and the limited frequency resolution, which could possibly explain the difference between the bump on the spectrum in Fig. 10 when $P_{\text {drive }}=55 \mathrm{~W}$ and the finite peaks on the spectrum in Fig. 5 when $i_{1} / Q_{\text {ext }}=0.0019$.

In summary, although there exists no analytical theory that is capable of accurately predicting magnetron behavior, the circuit model introduced in this paper is shown to be able to qualitatively recover the injection-locking characteristics observed in the experiment performed with the $\mathrm{cw}$ oven magnetron reflection amplifier.

\section{ACKNOWLEDGMENTS}

This research was supported by the Dual Use Science and Technology (DUST) under the Innovative Microwave Vacuum Electronics Multidisciplinary University Research Initiative (MURI). This research was funded by the Air Force Office of Scientific Research under Grant Nos. F49620-991-0297, F49620-00-1-0088 and F49620-02-1-0089.

\footnotetext{
${ }^{1}$ A. Pikovsky, M. Rosenblum, and J. Kurths, Synchronization (Cambridge University Press, Cambridge, 2001), p. 71.

${ }^{2}$ R. A. York and T. Itoh, IEEE Trans. Microwave Theory Tech. 46, 1920 (1998).

${ }^{3}$ J. S. Levine, J. Benford, B. Harteneck, and S. Montalvo, Proc. SPIE 1226, 44 (1990).

${ }^{4}$ J. S. Levine, N. Aiello, J. Benford, and B. Harteneck, J. Appl. Phys. 70, 2838 (1991).

${ }^{5} \mathrm{~W}$. M. White et al., Proceedings of the 32nd IEEE International Conference on Plasma Science, Monterey, CA, 18-23 June 2005 (unpublished), Paper 7B.5.

${ }^{6}$ S. C. Chen, IEEE Trans. Plasma Sci. 18, 570 (1990).

${ }^{7}$ S. C. Chen, G. Bekefi, and R. Temkin, Proc. SPIE 1226, 36 (1990).

${ }^{8}$ C. Chen, H. -W. Chan, and R. C. Davidson, Proc. SPIE 1407, 105 (1991).

${ }^{9}$ W. Woo, J. Benford, D. Fittinghoff, B. Harteneck, D. Price, R. Smith, and H. Sze, J. Appl. Phys. 65, 861 (1989).

${ }^{10}$ H. Sze, R. R. Smith, J. N. Benford, and B. D. Harteneck, IEEE Trans. Electromagn. Compat. 34, 235 (1992).

${ }^{11}$ J. Benford, H. Sze, W. Woo, R. R. Smith, and B. Harteneck, Phys. Rev. Lett. 62, 969 (1989).

${ }^{12}$ V. B. Neculaes et al., AIP Conf. Proc. 691, 301 (2003).

${ }^{13}$ G. L. Johnston, S. C. Chen, G. Bekefi, and R. C. Davidson, Proc. SPIE 1226, 108 (1990).

${ }^{14}$ J. M. Osepchuk, IEEE Trans. Microwave Theory Tech. 50, 975 (2002).

${ }^{15}$ W. C. Brown, A Reporting of Personal Observations of Noise in Magnetrons and CFAs (Raytheon, Massachusetts, 1988).

${ }^{16}$ R. Adler, Proc. IEEE 61, 1380 (1973).

${ }^{17}$ B. van der Pol, Proc. IRE 22, 1051 (1934).

${ }^{18}$ J. C. Slater, Microwave Electronics (Van Nostrand, New York, 1951), p. 190.

${ }^{19}$ J. E. Walsh, G. L. Johnston, R. C. Davidson, and D. J. Sullivan, Proc. SPIE 1061, 161 (1989).

${ }^{20}$ V. B. Neculaes, Ph.D. thesis, University of Michigan, 2005.

${ }^{21}$ P. Bogacki and L. F. Shampine, Appl. Math. Lett. 2, 1 (1989).

${ }^{22}$ J. R. Dormand and P. J. Prince, J. Comput. Appl. Math. 6, 19 (1980).

${ }^{23}{ }_{\text {MATLAB }}{ }^{\circledR}$ (The MathWorks, Inc., Natick, MA, 1984-2004).
} 